\title{
Leukocyte recognition algorithm in leucorrhea microscopic images based on ResNet-34 neural network
}

\author{
Xiuchao Wan ${ }^{1, *}$, Zhiyong Wang ${ }^{1}$, Guanghui Kong ${ }^{1}$, and Fengjun Xue ${ }^{2}$ \\ ${ }^{1}$ School of Electrical and Electronic Engineering, Tianjin University of Technology, Tianjin, China \\ ${ }^{2}$ Wuhan Running Education Research Institute, Wuhan, Hubei Province, China
}

\begin{abstract}
Automatic leucocyte recognition for leukorrhea microscopic images is a digital image processing technology in the field of machine learning. The existence and quantity of leukocytes in leucorrhea microscopic image is an important sign and basis to judge the inflammation of vagina or cervix. Therefore, the recognition and count of leucocyte is an effective means to evaluate the condition of the disease. To solve the problem of low efficiency of leucocyte recognition in traditional artificial microscopy, this paper proposes an automatic recognition algorithm based on ResNet-34 neural network. Firstly, Canny edge detection algorithm based on genetic algorithm is used to extract the foreground target in the leucorrhea microscopic image. Secondly, the leucocyte target is selected according to the connected region and boundary rectangle parameters of the foreground target. Finally, ResNet-34 neural network is applied for the classification of leukocytes. Experiments show that the recognition accuracy of leukocytes in leucorrhea microscopic image is $92.8 \%$, and the recall is $97.1 \%$, which is higher and better than other methods.
\end{abstract}

\section{Introduction}

With the continuous development of artificial intelligence technology, more and more medical automatic detection equipment is put into practice, bringing great convenience to hospitals and patients. The leucorrhea routine examination in the gynecological routine inspection is an important test to determine whether a woman's vagina is healthy. The inspection of leucorrhea cleanliness can determine the degree of contamination in the vagina. It is also able to assess the probability and degree of infection [1].

Leucorrhea routine examination methods currently used in hospitals are mainly based on conventional artificial microscopy, or microscopy supplemented with staining techniques $[2,3]$. However, traditional methods based on artificial microscopic examination highly rely on manual observation, which is time-consuming and laborious. Furthermore, they are also prone to be affected by some subjective factors. Therefore, traditional artificial microscopy has great limitations.

With the development of deep learning technology, researchers have proposed many algorithms for identifying elements such as cells in microscopic images. $\mathrm{Hu}$ Jingrong et al. propose a leukocyte recognition algorithm based on BP neural network and fuzzy clustering method. First, they segment leucocyte samples using Sobel edge detection, and then use BP neural network and fuzzy clustering to identify leukocytes [4]. Zhong $\mathrm{Ya}$ et al. propose a leucocyte recognition algorithm based on a convolutional neural network
$(\mathrm{CNN})$. First, the Otsu method is used to segment leucocyte samples, then they apply the CNN to recognize those segmented results [5]. Xiao Xuan et al. use a classification approach based on decision tree to classify the peripheral leukocytes. They extract some features, such as size, shape, intensity and color, as the input of decision tree for classification. [6]. A. Gautam et al. leverage the Otsu method for the segmentation of leukocytes. Further, only the nucleus is considered for feature extraction. Finally the Naïve Bayesian classifier is used for the classification of leukocytes [7].

Nevertheless, there are still some defects in above approaches, such as the non-ideal performance of binary images and the existence of edge discontinuities. As a result, while segmenting leukocytes, the segmentation accuracy is low and the missing rate is high. This is one of the significant reasons why the final recognition accuracy is difficult to achieve very high. To tackle these issues, we propose a Canny edge detection method based on genetic algorithm for leukocyte segmentation. The maximum entropy criterion is used to determine the threshold, and the optimal threshold pair is selected by using a genetic algorithm as the threshold pair for Canny edge detection to obtain a segmented image. Finally, ResNet-34 is used to classify the leukocyte samples obtained by segmentation to realize comprehensive and efficient leukocyte automatic recognition. Experiments show that our method achieves a much better performance than others.

\footnotetext{
* Corresponding author: 614479456@qq.com
} 


\section{Theory and method}

\subsection{Leukocyte segmentation method}

The size of leucorrhea microscopic images used in this study is $1440 \times 960$, as shown in Fig. 1 . The visible components in a leucorrhea microscopic image mainly include epithelial cells, leukocytes, bacilli, mixed bacterium and other impurities.

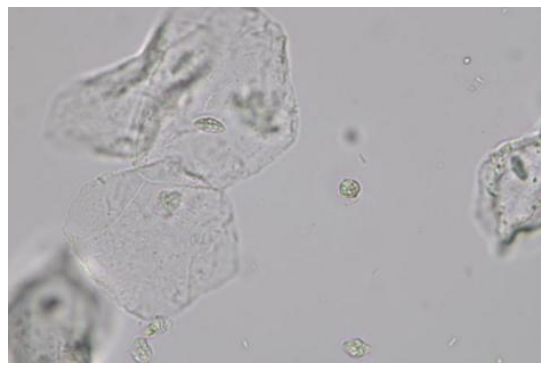

Fig. 1 Microscopic image of leucorrhea

In the leucorrhea microscopic image, the shape and size of the nucleus of the epithelial cells and impurities are similar to those of leukocytes. Therefore, the leukocytes and similar components in the leucorrhea microscopic image are segmented and labeled as positive samples and negative samples respectively through manual screening. In the positive samples, as shown in Fig. 2 (a), the leukocytes are polymorphonuclear neutrophils. Since each leaf of the nucleus presents a different connection state, the shapes, sizes and states of the leukocytes are different. In the negative samples, as shown in Fig. 2 (b), they contain the nucleus of epithelial cells, cell debris, mixed bacterium and other tangible substances, whose shapes, sizes and states are also different with each others'.
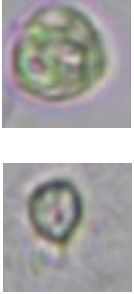
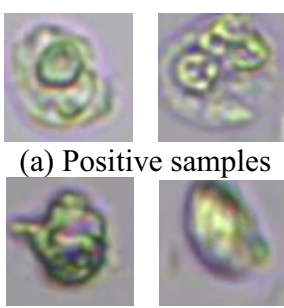

(b)Negative samples
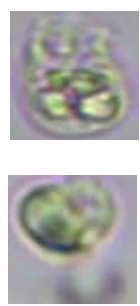

Fig. 2 Split samples

The algorithms most researchers commonly apply for leucocyte segmentation include Otsu method, watershed segmentation algorithm, edge detection algorithms, and convolutional neural network, etc. [8-11]. We propose a Canny edge detection method based on genetic algorithm for leukocytes segmentation, aiming to solve the problems that leucorrhea microscopic images usually have weak edges, and the variance of grayscale is not obvious.

First, we convert the leukorrhea images into grayscale. Then we use adaptive median filtering to remove the noise contained in the grayscale image, and reduce its interference and influence on subsequent morphological processing. Secondly, we use the top-hat transformation to enhance the edge part of the image. Then the Canny edge detection algorithm based on genetic algorithm is used to extract edges and get the binary image, as shown in Fig. 3 (b). By replacing the gradient calculation template in the traditional Canny edge detection algorithm with a Sobel operator template, it is able to calculate the gradient direction and amplitude while suppressing noise effectively. The threshold is selected using maximum entropy criterion in Canny edge detection algorithm. Furthermore, we apply the genetic algorithm to choose the optimal threshold pairs as the high and low threshold of connected edges. Afterward a circular structure element with a radius of 5 is used to perform a morphological close operation to fill the holes in the binary image. The result is shown in Fig. 3 (c).

Finally, the connected regions are labeled on the binary image after the close operation, and the area of each connected region and its boundary rectangle parameters are calculated. According to the parameters of the connected regions and their boundary rectangles, we select the connected areas that meet the leukocytes for labeling. The binary image after processing is shown in Fig. 3(d).
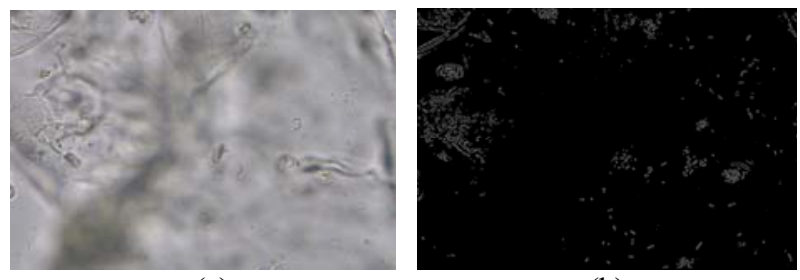

(a)

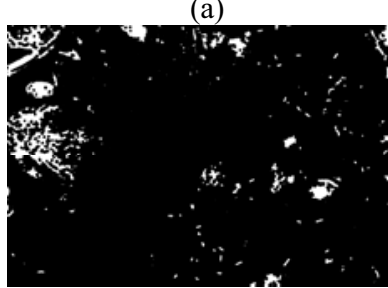

(c)

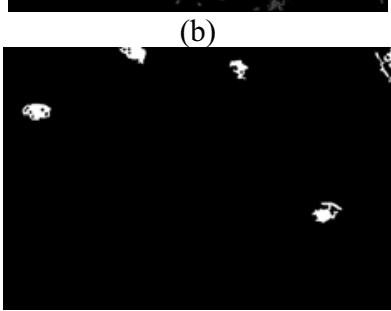

(d)
Fig. 3 Image processing

(a) Original image (b) Canny edge detection image (c) Image after close operation (d) The labeled binary image

\subsection{Leukocyte recognition method}

With the continuous development of convolutional neural networks, the network structure goes deeper and deeper. As a result, some issues occur inevitably, such as the vanishing gradient problem, the exploding gradient problem and the network degradation.

The vanishing gradient problem and the exploding gradient problem are caused by the special structure of neural networks and special method of calculating parameters. Network degradation refers to increasing the number of layers of the neural network but the effect is not significantly improved. This means that there exist some redundant layers in the network. They are not able to help with training, but interfere those well trained parameters. 
In order to solve the above problems, Kaiming He et al. proposed the ResNet (Deep Residual Network) architecture in 2015 [12]. The main idea of ResNet is to add a shortcut connection in the network (i.e. Highway Network). ResNet merges several convolutional layers into a residual block, as shown in Fig. 4.

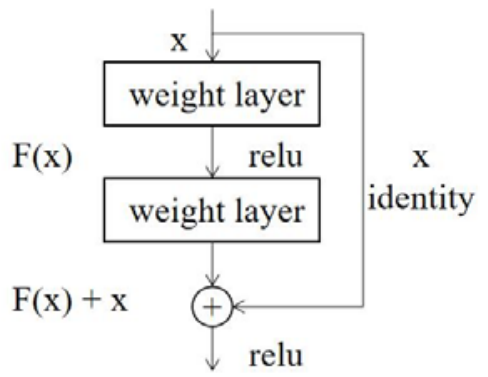

Fig. 4 Residual block structure
ResNet solves the problem of information loss during information transmission. Compared with traditional method of learning the mapping function $H(x)$, it turns to learn the residual function $F(x)=H(x)-x$ instead. By passing the input directly to the output, the integrity of the information is protected. In addition, the simplification of learning goals also reduces the difficulty of learning. In order to identify the segmented sample cells faster and more accurately, we use the ResNet-34 network as the recognizer. The ResNet-34 network structure is shown in Fig. 5.

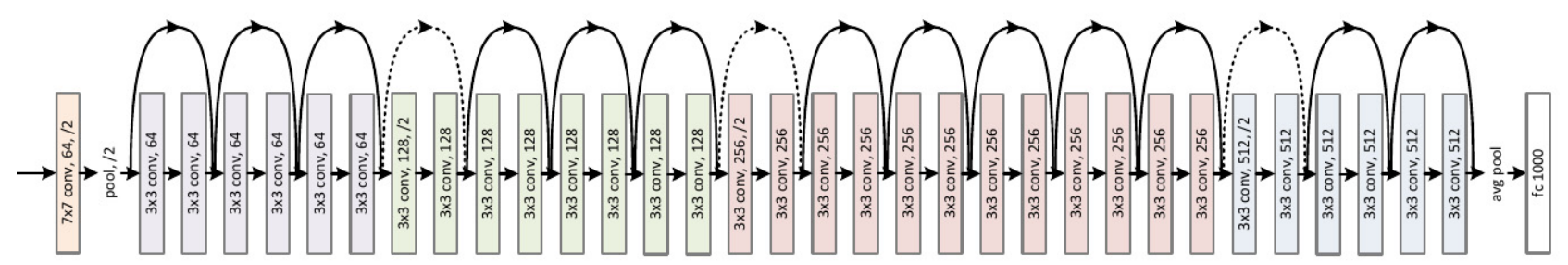

Fig. 5 Resnet34 network architecture

Since the segmented leukocyte images have different sizes, they should be scaled to be ready for the input of ResNet-34. Bilinear interpolation is performed on the segmented sample cell images to resize them uniformly into an image of size $60 \times 60$.

\subsection{Algorithm evaluation method}

In this paper, We use three evaluation criteria, which are accuracy, recall and F1-score to objectively validate the performance of segmentation and recognition. These criteria are shown as follows.

$$
\begin{gathered}
\text { Accuracy }=\frac{T P+T N}{T P+F P+T N+F N} \\
R \text { ecall }=\frac{T P}{T P+F N} \\
F 1=\frac{2 A \text { ccuracy } \times \text { Recall }}{A \text { ccuracy }+ \text { Recall }}
\end{gathered}
$$

\section{Experimental results and analysis}

In this experiment, we collect 222 leucorrhea microscopic images, each of which has 31 views. So there are 6882 sample images in total. We select 3000 samples for our experiment by manual filtering and labeling.

\subsection{Segmentation results and analysis}

The results of segmenting the leukocytes in the leucorrhea microscopic image by the Canny edge detection algorithm based on genetic algorithm are shown in Fig. 6 (a) (c) (e). As a comparison, the segmentation results based on Otsu method proposed in Reference [6] are shown in Fig. 6 (b) (d) (f).

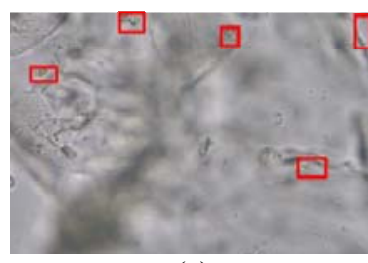

(a)

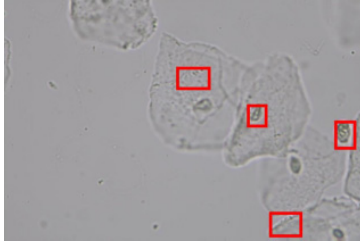

(c)

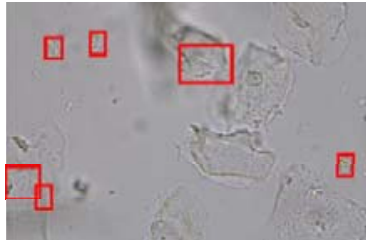

(e)

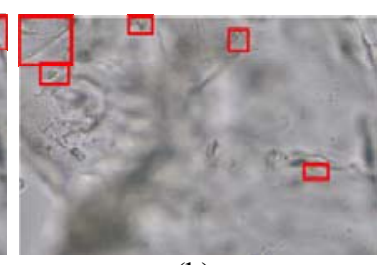

(b)

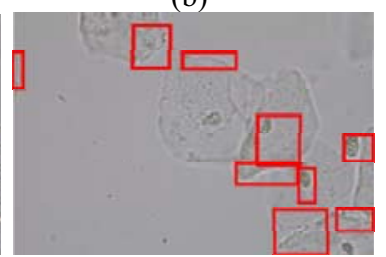

(d)

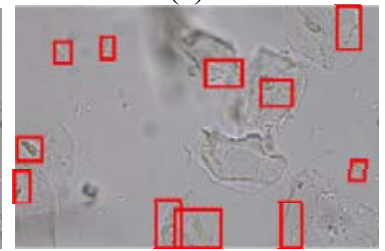

(f)
Fig. 6 Segmentation results

(a) (c) (e) Segmentation results of the algorithm in this paper (b) (d) (f) Reference [6] Algorithm segmentation results

We can observe from Fig. 6 (b) (d) (f) that the method of Ref. [6] will produce a lot of other tangible compositions besides the leukocytes. The number of true positive samples (TP), true negative samples (TN), the false negative samples (FN), and the recall of two methods are calculated separately, which are shown in Table 1. 
Table 1. Leukocyte segmentation results.

\begin{tabular}{|c|c|c|c|c|}
\hline Methods & TP & TN & FN & Recall/\% \\
\hline Reference [6] & 4038 & 2386 & 938 & 81.1 \\
\hline Our method & 4914 & 1528 & 121 & 97.6 \\
\hline
\end{tabular}

Comparing the segmentation results of Otsu method in Ref. [6], we can find that the result of Otsu method contains too many non-leukocyte parts. Moreover, for leucorrhea microscopic images which containing leucocyte adhering to other tangible compositions, the accuracy of our segmentation method is higher.

\subsection{Recognition results and analysis}

In our classification experiment, we choose 1500 true positive samples and 1500 true negative ones as the original dataset of ResNet-34 for recognition. They are randomly divided into 10 groups for 10 -fold cross validation. The result is evaluated by the average of 10 test cases. We compare our experimental results with Ref.[5] and Ref.[6], as shown in Table 2.

Table 2. Leukocyte recognition results.

\begin{tabular}{cccc}
\hline Methods & Recall/\% & Accuracy/\% & F1/\% \\
\hline Reference [5] & $95.3 \pm 1.8$ & $90.0 \pm 2.3$ & $92.5 \pm 2.05$ \\
\hline Reference [6] & $95.0 \pm 1.3$ & $89.5 \pm 1.5$ & $92.1 \pm 1.4$ \\
\hline Our method & $97.1 \pm 0.8$ & $92.8 \pm 0.7$ & $94.9 \pm 0.75$ \\
\hline
\end{tabular}

It can be seen from the table that using ResNet-34 as a recognizer, the effect is better than that in Ref. [5] and Ref. [6] in terms of accuracy and recall.

As an experimental conclusion, the recall of Canny edge detection based on genetic algorithm is $97.6 \%$, which indicates that our method can segment all leukocytes in leucorrhea as much as possible. It can also achieve a good segmentation performance for the problem of cell adhesion. The accuracy of leucocyte recognition based on ResNet-34 neural network is $92.8 \%$, and the recall is $97.1 \%$, which is higher in efficiency and better in effect than convolutional neural network and BP neural network used in Ref.[5] and Ref. [6]. We show our recognition performance in Fig. 7. In the figure, leukocytes are marked in the red rectangle while negative samples are marked in the blue rectangle.

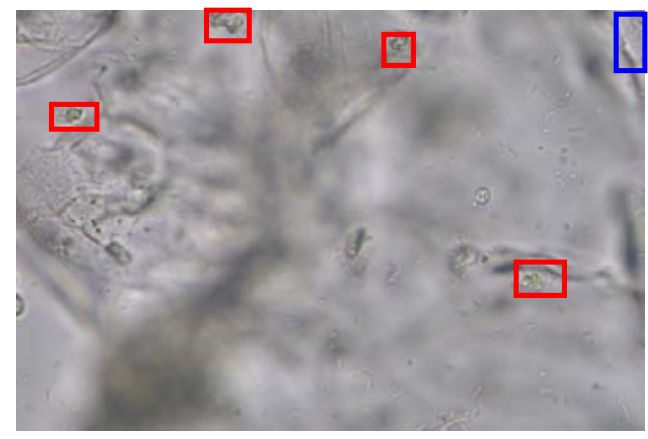

Fig. 7 Leukocyte recognition results

\section{Conclusion}

Detection of leukocytes in leucorrhea is a simple and effective method to evaluate inflammation of vagina or cervical tissue. Aiming at the requirement of automatic recognition of leukocytes in leucorrhea microscopic images, we propose an automatic leukocyte recognition algorithm based on ResNet-34 neural network. The algorithm mainly includes two main sections. The first is Canny edge detection algorithm based on genetic algorithm. This algorithm can hugely improve the performance of segmenting the leukocytes as well as ensuring a low missing rate. The second is to train a ResNet-34 neural network as a recognizer. It not only avoids the complex procedure of extracting features manually in traditional algorithms, but also alleviates the vanishing gradient problem, the exploding gradient problem and the network degradation. To realize the automatic recognition of leukocytes in leukorrhea using image processing and machine learning method can not only reduce the workload of conventional inspection of traditional artificial leucorrhea, but also reduce the subjective impact, thereby improving efficiency and accuracy.

\section{References}

1. Y.-Q. Li. "Analysis of the results and countermeasures of 600 cases of leucorrhea in women." World Latest Medical Information Abstracts (Electronic Edition). 18, 171-171, (2018)

2. G.-N. Ding. "A comparative analysis of three gynecological leucorrhea examination methods." China Journal of Modern Medicine,13, 52-53, (2011)

3. X.-W. Ling, et al. "Comparison and analysis of the multi-detection results of leucorrhea routine with 2 methods." Chinese Journal of Health Laboratory Technology,25,2136-2138.(2015)

4. J.-R. Hu. Research on Intelligent Recognition of Shaped Components in Microscopic Images of Leucorrhea. University of Electronic Science and Technology of China. 2017

5. Y. Zhong, J. Zhang, J. Xiao. “Automatic Detection of Leukocytes in Leucorrhea Based on Convolution Neural Network." Chinese Journal of Biomedical Engineering, 37, 163-168. (2018)

6. X. Xuan, Q. Liao, K.-A. Jiang. "A novel decisiontree based classification of white blood cells." SPIE, 5747, 1120-1127. (2005)

7. A Gautam, P. Singh, B. Raman and H. Bhadauria, "Automatic classification of leukocytes using morphological features and Naïve Bayes classifier," 2016 IEEE TENCON, 1023-1027. (2016)

8. Q. Chen, L. Zhao, J. Lu, G. Kuang, N. Wang and Y. Jiang, "Modified two-dimensional otsu image segmentation algorithm and fast realisation," IET Img Pro, 6, 426-433, (2012)

9. Q. Sun, F. Liang, F. Wang, H. Cong, Q. Li and J. Yan, "Investigation on the Geometrical 
Characteristics of Secondary Arc by Image Edge Detection," IEEE Transactions on Plasma Science, 46, 2016-2025, (2018)

10. Cai, Q, et al. "A Watershed Image Segmentation Algorithm Based on Self-adaptive Marking and Interregional Affinity Propagation Clustering." 45, 1911-1918, (2017)

11. Hiramatsu, et al. "Cell Image Segmentation by Integrating Multiple CNNs." IEEE/CVF CVPRW, 2286-22866. (2018)

12. K.-M. He, et al. "Deep Residual Learning for Image Recognition.’'IEEE CCVPR, 1, 770-778, (2016) 